\title{
ADVANCED TECHNOLOGY LUNAR ASTRONOMY MISSION, THE MOON AS AN IMMENSE OPTICAL BENCH IN VACUUM
}

\author{
PETER C. CHEN, YOJI KONDO AND RONALD J. OLIVERSEN \\ Goddard Space Flight Center \\ Greenbelt, MD 20771, USA
}

The Moon combines some of the most attractive features for astronomical observations from space (no atmospheric absorption, perfect seeing, etc.) and those from ground (large steady optical bench, ease of control from Earth, etc.). Astronomers have planned for telescopes on the Moon for decades but, due to its primary obstacle - high cost - they have not yet been built, save one small telescope that was carried to the Moon on an Apollo mission.

However, recent technological developments have brought the concept of lunar telescopes within our financial reach - as will be shown below. Instead of a billion dollar telescope, we are looking at a program in the range of several tens of millions of dollars in the price range of the NASA MIDEX Program.

Consider the following changes since the study of the Lunar Transit Telescope conducted at the NASA Marshall Space Flight Center in 1991-94. For example, the areal density of the mirror used in the 1991-94 study was $30 \mathrm{~kg}$ per square meter, whereas ultra-lightweight optics we are developing have an areal density ten times lighter at $2 \mathrm{~kg}$ per square meter. We will be using High Temperature Superconductor (HTS) bearings for telescope drives; these are non-contact and do not wear out. The HTS operates at a temperature in the range of about 90 degrees $\mathrm{K}$ and can be radiatively cooled.

$$
\text { 1991-94 Present }
$$

$\begin{array}{lcc}\text { Mirror Mass } & 25 \mathrm{~kg} & 3 \mathrm{~kg} \\ \text { Support Structure } & 150 \mathrm{~kg} & 15 \mathrm{~kg} \\ \text { Payload } & 400 \mathrm{~kg} & 25 \mathrm{~kg} \\ \text { Lander } & 2000 \mathrm{~kg} & 20 \mathrm{~kg} \text { (est.) }\end{array}$

Rocket Mission Cost US $\$ 1 \mathrm{~B} \quad$ US $\$ 20-40 \mathrm{M}$

Other innovations include the development of Charge Injection Detectors (CID), which are silicon based solid state array sensors that can withstand solar flares for decades with minimum shielding. We also propose to use a low cost agile missile interceptor known as the LEAP (Light Exo-Atmospheric Projectile) as a small payload lunar lander at a cost of only about US $\$ 50 \mathrm{~K}$.

The most cost effective lunar interferometer in the near future would probably be a dilute synthetic aperture optical interferometer with one kilometer baseline (a limit imposed by the natural curvature of the Moon). This baseline corresponds to a resolution of 0.1 milliarcsecond in the visible light, or more than a hundred times that of the Hubble Space Telescope. Using a suitable crater, this baseline could be extended significantly. There also exist alternative means for expanding the baseline, such using high struts.

An array of some six 1-meter telescopes will be needed but such an interferometer array is probably within the capabilities of a medium class launcher such as the US Delta or the Japanese M-5 (730 kg and $520 \mathrm{~kg}$, respectively, to the Moon). Following the idea of Layberie, the individual telescopes can be counted on a rover to move around continuously during observation. This would 
permit path length equalization for the beams and would eliminate the need for optical delay lines.

A more ambitious idea would involve the use of the Japanese $\mathrm{H} 2$ rocket. The $\mathrm{H} 2$ can carry 2,800 $\mathrm{kg}$ to the Moon and can accommodate apertures of up to $4 \mathrm{~m}$. This raises the possibility of a truly spectacular optical interferometer array far surpassing any set of telescopes anywhere on Earth or in space that are currently being envisaged.

\section{References}

- "Mission to the Moon: Europe's Priorities for the Scientific Exploration and Utilization of the Moon." Report of the Lunar Study Steering Group. ESA SP-1150, June 1992. Chapter 2, pp40-42. 